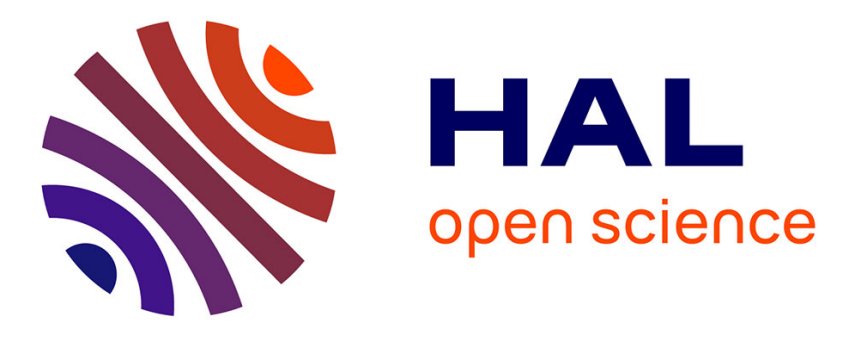

\title{
Verification of class liveness properties with Java modeling language
}

Alain Giorgetti, Julien Groslambert, Jacques Julliand, Olga Kouchnarenko

\section{To cite this version:}

Alain Giorgetti, Julien Groslambert, Jacques Julliand, Olga Kouchnarenko. Verification of class liveness properties with Java modeling language. IET Software, 2008, 2 (6), pp.500-514. 10.1049/ietsen:20080008 . hal-00561340

\section{HAL Id: hal-00561340 https://hal.science/hal-00561340}

Submitted on 1 Feb 2011

HAL is a multi-disciplinary open access archive for the deposit and dissemination of scientific research documents, whether they are published or not. The documents may come from teaching and research institutions in France or abroad, or from public or private research centers.
L'archive ouverte pluridisciplinaire HAL, est destinée au dépôt et à la diffusion de documents scientifiques de niveau recherche, publiés ou non, émanant des établissements d'enseignement et de recherche français ou étrangers, des laboratoires publics ou privés. 


\title{
Verification of Class Liveness Properties with JML*
}

\author{
A. Giorgetti ${ }^{1,2}$, J. Groslambert ${ }^{3}$, J. Julliand ${ }^{2}$ and O. Kouchnarenko ${ }^{1,2}$ \\ \{giorgett, julliand, kouchna\}@lifc.univ-fcomte.fr \\ Julien.Groslambert@trusted-labs.com \\ ${ }^{1}$ INRIA / CASSIS \\ ${ }^{2}$ LIFC / University of Franche-Comté $\quad{ }^{3}$ Trusted Labs \\ 16 route de Gray 5 rue du Bailliage \\ F-25030 Besançon Cedex F-78000 Versailles
}

\begin{abstract}
Static checking is key for the security of software components. As a component model, this paper considers a Java class enriched with annotations from the Java Modeling Language (JML). It defines a formal execution semantics for repetitive method invocations from this annotated class, called the class in isolation semantics. Afterwards, a pattern of liveness properties is defined, together with its formal semantics, providing a foundation for both static and runtime checking. This pattern is then inscribed in a complete language of temporal properties, called JTPL (Java Temporal Pattern Language), extending JML. We particularly address the verification of liveness properties by automatically translating the temporal properties into JML annotations for this class. This automatic translation is implemented in a tool called JAG (JML Annotation Generator). Correctness of the generated annotations ensures that the temporal property is established for the executions of the class in isolation.
\end{abstract}

\section{Introduction}

Component-based development provides significant advantages - portability, adaptability, re-usability, etc. - when developing, e.g., Java Card smart card applications [6] or when composing Web services within Service Component Architecture (SCA) - a relatively new initiative advocated by users of Java technology. In this framework, the use of components of distributed applications or component-based applications

*Research partially funded by the French National Research Agency, ANR-06-SETI-017 TACOS. 
necessitates ensuring not only invariance and safety properties but also partial correctness and liveness properties of components. We consider a component modeled by a Java class that is annotated in Java Modeling Language (JML for short).

Currently, more and more tools aiming at the verification of Java programs are adopting JML as property specification language (see [7] for an overview). JML ${ }^{1}$ is a specification language syntactically and semantically close to Java, thus making specifications more accessible to Java programmers. JML allows adding basic formal annotations - like method pre- and post-conditions or invariants - to the Java class, thus proposing a way to modularly verify Java applications. However, it is difficult to directly specify complex dynamic properties in JML, like temporal properties [17], that are often needed to express the security policies that the Java implementation has to ensure. Therefore, Huisman and Trentelman [28] proposed a language of temporal properties - later called JTPL, for Java Temporal Pattern Language [14].

Our main purpose is to verify liveness properties of Java/JML components using a JML extension. The first contribution is a formal execution semantics for repetitive method invocations from this component, called the class in isolation semantics. To infer class invariants by abstract interpretation, Logozzo [21] proposed a semantics of partial execution paths of an object-oriented program and of a so-called class in isolation. Our work follows this approach but, for verifying liveness properties, we define a complete execution path semantics of a class in isolation. Moreover, since we consider Java/JML components, we take into account the JML semantics to define the class in isolation semantics. The second contribution is an extension of the JML type specifications with a temporal specification of liveness by introducing a new specification clause in Java classes - called the liveness clause. A deep integration of this liveness clause in JML is achieved by using the same semantics of visible states as for JML invariant and constraint clauses. The third contribution is a verification method for liveness properties by generating JML invariants and history constraints. The fourth contribution is a systematic translation of temporal properties into JML annotations. Thanks to the semantics, we establish the correctness of the translation. Notice that the second and third contributions were presented in [15] without proofs nor explicit examples. To make it short, the main extensions to [15] are (1) a complete formal semantics for the executions of a Java/JML component, and (2) the translation of all the liveness formulas of the JTPL temporal logic into standard JML annotations.

This paper is organised as follows: Section 2 quickly presents JML on an example. Section 3 introduces the mathematical background used in the next sections. Section 4 presents the semantic framework of the paper. In particular, it defines a semantics for a class in isolation and gives the semantics of JML main annotations. Moreover, the semantics of visible states is recalled and formalised (upon an

\footnotetext{
${ }^{1}$ See http://www. jmlspecs.org.
} 
ad'hoc semantics of a Java class). Next, Section 5 defines the liveness clause and its formal semantics. Section 5 also presents the verification of liveness properties on a class in isolation through appropriate annotation generation. Section 6 presents the application of the annotation generation method to the JTPL temporal liveness properties based on their translation into the liveness clause that we propose to extend JML. Section 7 presents the JAG tool implementing this automatic generation of annotations. Section 8 concludes by giving some perspectives and future work.

\section{Overview of JML and Example}

JML (Java Modeling Language) [18] is a specification language especially tailored for Java applications. Originally, JML was proposed by G.T. Leavens and his team; the development of JML is now a community effort. JML has been successfully used in several case studies to specify Java applications, and more especially to specify smart card applications $[6,16]$. JML is developed following the Design by Contract approach [23], where classes are annotated with class invariants and method pre- and post-conditions. The predicates are side-effect free boolean Java expressions, extended with specific constructs. Specifications are written as Java comments marked with an $@$, i.e., annotations follow //@ or are enclosed between /*@ and @*/. Figure 1 presents some JML annotations on the simple example of a buffer.

The class Buffer works as follows: a method storeData() customises the application by setting the transaction length. Then, one can initialise a new transaction with the method begin(), creating a new temporary buffer. Afterwards, a write() method fills the modifications in the temporary buffer that is validated, i.e., assigned to the attribute status, by an invocation of commit(). It is also possible to abort the transaction by an invocation of the method abort().

Figure 1 displays a class invariant, i.e. a predicate that has to hold on every so-called JML visible state. History constraints allow expressing a relation between the pre- and post-state of all methods. Pre-state values of expressions are denoted by the JML keyword \old. Using the clause for, one may specify the methods list for which the history constraint must be satisfied. When this clause is omitted, the constraint must hold for all the class methods. The clause requires denotes the pre-condition of the method, i.e., a predicate that must be true when the method is called. A post-condition is expressed with an ensures clause. A method may exceptionally terminate by throwing an exception and satisfying the exceptional post-condition (signals clause). The method specification can also contain a diverges clause (not displayed in this example). If the predicate of a diverges clause of a method $m$ is satisfied by the pre-state of $m$, then the execution of $m$ may not terminate. Otherwise the method must terminate. By default, the JML diverges clause is set to false. JML also introduces its own variables - declared with 
the keyword ghost. A special set annotation exists to assign their value. For instance, trDepth $=$ true means that a transaction is in progress. This variable allows expressing that every opened transaction must eventually be closed. This is an example of liveness property that will be translated into a set of JML annotations. The correctness of a Java class w.r.t. JML annotations can be established by modelchecking [26] or by a prover (B or Coq) via a proof obligation generator (Jack [8] or Krakatoa [22]).

\section{Preliminaries}

This section introduces some definitions and notations used in the other sections. It recalls the notion of sequence and some useful results for the existence of fixpoints in lattices.

\subsection{Notations}

Familiarity with basic set theory is assumed. Given a binary relation $R \subseteq S_{1} \times S_{2}, \operatorname{dom}(R)$ is its domain, $\operatorname{ran}(R)$ is its range and $R^{-1}$ is the inverse relation. If $\operatorname{dom}(R)=S_{1}$ then the relation is total. A relation $f \subseteq S_{1} \times S_{2}$ is a partial function from $S_{1}$ to $S_{2}$, denoted $S_{1} \rightarrow S_{2}$, if each element of its domain has a single image. It is a (total) function, denoted $S_{1} \rightarrow S_{2}$, if it is total and a partial function. An endofunction of $S$ is a function from $S$ to itself. For any function $f: S_{1} \rightarrow S_{2}, x \in S_{1}$ and $y \in S_{2}$, the update of $f$ with $y$ at $x$, denoted $f[x \mapsto y]$ is the unique function such that:

$$
f[x \mapsto y](u)= \begin{cases}f(u) & \text { if } u \neq x \\ y & \text { if } u=x\end{cases}
$$

More generally, we write $f\left[x_{1} \mapsto y_{1}, \ldots, x_{n} \mapsto y_{n}\right]$, instead of $f\left[x_{1} \mapsto y_{1}\right] \ldots\left[x_{n} \mapsto y_{n}\right]$, when the $x_{1} \ldots x_{n}$ are all different.

\subsection{Sequences}

Let $S$ be a (nonempty) set. A sequence is a partial function $\sigma$ from $\mathbb{N}$ to $S$ such that the set $\operatorname{dom}(\sigma)$ is either $\mathbb{N}$ or a finite subset $[0, \ldots, k]$ for some $k$ in $\mathbb{N}$. The empty sequence, whose domain is the empty set, is denoted $\epsilon$. A sequence $\sigma$ is infinite if $\operatorname{dom}(\sigma)=\mathbb{N}$, finite otherwise. The length $\operatorname{len}(\sigma)$ of a sequence $\sigma$ is $n$ if it is finite and if $\operatorname{dom}(\sigma)=[0, \ldots, n-1], \omega$ otherwise. The last element last $(\sigma)$ of a sequence $\sigma$ is $\sigma(\operatorname{len}(\sigma)-1)$ if this sequence is finite and nonempty, $\omega$ otherwise. We use $S^{*}, S^{+}, S^{\omega}, S^{* \omega}$ and $S^{+\omega}$ to respectively denote the sets of finite, nonempty finite, infinite, finite or infinite, and nonempty finite or infinite sequences. 
The concatenation $\alpha . \beta$ of two sequences $\alpha, \beta \in S^{* \omega}$ of length $l=\operatorname{len}(\alpha)$ and $m=\operatorname{len}(\beta)$ is a sequence of length $\operatorname{len}(\alpha . \beta)=l \oplus m$, where $\oplus$ extends the addition of $\mathbb{N}$ to $\mathbb{N} \cup\{\omega\}$, with $\omega \oplus n=n \oplus \omega=\omega$ for any $n \in \mathbb{N} \cup\{\omega\} . \sigma=\alpha . \beta$ is defined by $\sigma(i)=\alpha(i)$ for $0 \leq i<l$ and $\sigma(i)=\beta(i-l)$ for $l \leq i<l e n(\sigma)$. Concatenation of sequences extends to sets of sequences in a standard way, with the same notation.

Two nonempty sequences $\alpha, \beta \in S^{+\omega}$ of length $l=\operatorname{len}(\alpha)$ and $m=\operatorname{len}(\beta)$ are joinable iff last $(\alpha)$ is $\beta(0)$ or $\omega$. When they are joinable, their join (or junction) $\alpha^{\wedge} \beta$ is a sequence $\sigma$ of length $\operatorname{len}(\sigma)=(l \oplus m) \ominus 1$, where $\ominus$ extends the subtraction of $\mathbb{N}$ to $\mathbb{N} \cup\{\omega\}$, with $\omega \ominus n=\omega$ for any $n \in \mathbb{N} \cup\{\omega\} . \sigma=\alpha^{\frown} \beta$ is such that $\sigma(i)=\alpha(i)$ for all $0 \leq i<l$ and $\sigma(i)=\beta(i-l+1)$ for all $l \leq i<\operatorname{len}(\sigma)$ when $l<\omega$. The junction $S^{\curvearrowleft} T$ of the sets of nonempty sequences $S$ and $T$ is the set of junctions $\alpha^{\curvearrowleft} \beta$ of joinable sequences $\alpha \in S$ and $\beta \in T$.

\subsection{Complete Lattices}

A partial order $\sqsubseteq$ on a set $S$ is a relation on $S$ which is reflexive $(\forall x \in S . x \sqsubseteq x)$, transitive $(\forall x, y, z \in$ $S .(x \sqsubseteq y \wedge y \sqsubseteq z) \Rightarrow x \sqsubseteq z)$ and antisymmetric $(\forall x, y \in S .(x \sqsubseteq y \wedge y \sqsubseteq x) \Rightarrow x=y)$. A partially ordered set $\langle S, \sqsubseteq\rangle$, or poset, is a set equipped with a partial order $\sqsubseteq$. A lower bound $l$ of $U \subseteq S$ is an element $l$ of $S$ such that $\forall x \in U . l \sqsubseteq x$. A greatest lower bound of $U$ is a lower bound $g$ of $U$ such that $l \sqsubseteq g$ holds for all lower bound $l$ of $U$. A (least) upper bound of $U$ for $\sqsubseteq$ is a (greatest) lower bound of $U$ for the inverse partial order $\sqsubseteq^{-1}$. By antisymmetry of $\sqsubseteq$, greatest lower and least upper bounds, when they exist, are unique.

A complete lattice $\langle S, \sqsubseteq, \sqcup, \sqcap\rangle$ is a poset $\langle S, \sqsubseteq\rangle$ where every subset $U \subseteq S$ has a least upper bound, denoted $\sqcap U$, and a greatest lower bound, denoted $\sqcup U$. An endofunction $f$ of $S$ is monotone if $\forall x, y \in$ S. $x \sqsubseteq y \Rightarrow f(x) \sqsubseteq f(y)$. A consequence of Tarski's fixpoint theorem [27] is the existence of least and greatest fixpoints for any monotone function in a complete lattice.

Proposition 1 Every monotone endofunction $f$ on a complete lattice $\langle L, \sqsubseteq, \sqcup, \sqcap\rangle$ admits a least fixpoint lfp $(f)=_{\operatorname{def}} \sqcap\{x \mid x \in L \wedge f(x) \sqsubseteq x\}$ and a greatest fixpoint $g f p(f)=_{\text {def }} \sqcup\{x \mid x \in L \wedge x \sqsubseteq f(x)\}$.

\subsection{Sequence Set Lattice}

When a program can either run forever or end, its execution (or trace) semantics is a set of finite or infinite sequences (of states). Following [10], these sets can be specified as fixpoints in the set $2^{S^{+\omega}}$ of sets of nonempty finite or infinite sequences. The following proposition defines a lattice over this set by fusion of the complete lattices $\left\langle 2^{S^{+}}, \subseteq, \cup, \cap\right\rangle$ and $\left\langle 2^{S^{\omega}}, \supseteq, \cap, \cup\right\rangle$ of sets of respectively nonempty finite 
and infinite sequences. A proof that they are complete lattices can be found in [10], Th. 11 and 12 . In all that follows, $X^{+}$(resp. $X^{\omega}$ ) shortens $X \cap S^{+}$(resp. $X \cap S^{\omega}$ ) for any $X$ in $2^{S^{+\omega}}$.

Proposition 2 (Corollary of [10], Th. 9) Let $2^{S^{+\omega}}$ be the (disjoint) union of $2^{S^{+}}$and $2^{S^{\omega}}$. For any $X$ in $2^{S^{+\omega}}$, let $\sqsubseteq$ be defined by $X \sqsubseteq Y=X^{+} \subseteq Y^{+} \wedge X^{\omega} \supseteq Y^{\omega}$. For any subset $Z$ of $2^{S^{+\omega}}$, let $\sqcup$ and $\sqcap$ be respectively defined by $\sqcup Z=\bigcup_{X \in Z} X^{+} \cup \bigcap_{X \in Z} X^{\omega}$ and $\sqcap Z=\bigcap_{X \in Z} X^{+} \cup \bigcup_{X \in Z} X^{\omega}$.

Then $\left\langle 2^{S^{+\omega}}, \sqsubseteq, \sqcup, \sqcap\right\rangle$ is a complete lattice.

\section{Execution Semantics}

Our aim is to verify liveness properties of Java/JML components. A suitable semantics for this is a set of maximal execution paths. Intuitively, an execution path (or simply an execution) is a sequence of states reached during an execution of the class. An execution path is maximal if it cannot be extended to form a longer execution path. A maximal execution path is either infinite or is terminating with a blocking state.

\subsection{Context Restrictions}

We study a component that is a Java class enriched with some JML annotations: invariant, constraint and ghost variables for the class, behavior for methods and set in their bodies. The annotation pure means that a method is side-effect free. The annotations helper and assignable are useless in defining the liveness properties that we address. Consequently, we do not take these annotations into account. We do not address the problems of inheritance, multithreading and exception hierarchy. To simplify the presentation, we do not take into account the finalizers and the static methods. The execution of the component environment is restricted to creating only one instance of the class. The execution invokes only the non static methods.

We assume that the environment and the class respect the contract defined by the JML specifications. That means that the environment calls method $m$ from a memory state that satisfies its requires condition. It is assumed that the annotated class is consistent, i.e. each method $m$ leads to a state that satisfies either its ensures condition if $m$ does not diverge and does not raise an exception or the signals predicate if it raises an exception. 


\subsection{Java Subset Semantics}

In this section Definitions 1 and 2 describe the Java/JML components that we consider. Then an execution semantics of a Java subset is given in Def. 4 as a sequence of memory states, defined in Def. 3.

A component is a Java class defining a set of methods and a set of attributes and ghost variables. The class can be annotated with JML annotations as invariant, constraint and behavior. A behaviour is a method annotation. A class can also contain ghost variables and set annotations. A component is, therefore, an annotated class in Java/JML defined as follows:

Definition 1 (Annotated Class) An annotated class $C$ is a tuple $\left(V_{C}, I_{C}, C_{C}, M_{C}\right)$ where $V_{C}$ is the set of attributes and ghost variables of the class, $I_{C}$ is a set of $J M L$ invariants, $C_{C}$ is a set of JML constraints and $M_{C}$ is the set of all method names of the class except the constructor $i_{C} . A$ method

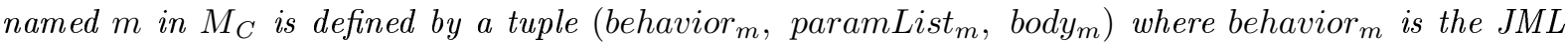
specification of a canonical behaviour, paramList ${ }_{m}$ is its set of parameters and body is $_{\text {in }}$ the Java program that implements $m$.

By a desugaring operation [25], the method $m$ behaviours can be reduced to a single canonical behaviour annotation: behavior; requires $P_{m}$; diverges $D_{m}$; ensures $Q_{m}$; signals (Exception e) $R_{m}$;. In the rest of the paper, $P_{m}, D_{m}, Q_{m}$ and $R_{m}$ respectively denote the requires, diverges, ensures and signals predicates of the behaviour of method $m$.

The addressed Java subset to define method bodies bodym is composed of atomic and method call statements respectively denoted by as and $m(E, \ldots, E)$, sequential, conditional and iterative statement compositions, and exception handling. An atomic statement is any statement that does not define other memory states than the states before and after its execution. A typical example is an assignment of a variable in $V_{C}$.

Definition 2 (Java Subset) Let $E$ be a Java expression, $m$ a Java identifier and $P$ a Java predicate (a boolean Java expression). We consider the Java statement subset $T$ defined by the following abstract syntax:

$T::=$ as $|m(E, \ldots, E)| T ; T \mid$ if $(P)\{T\}$ else $\{T\} \mid$ while $(P)\{T\}$

| throw $\mid \operatorname{try}\{T\}$ catch $\{T\} \mid \operatorname{try}\{T\}$ finally $\{T\}$.

A memory state assigns values to variables. For a component in isolation, we consider three sets of variables, namely: the set $V_{C}$ of attributes and ghost variables, the set $P_{C}=\bigcup_{m \in M_{C}}$ paramList $_{m}$ of parameters of all the methods (to simplify, we assume that distinct methods have disjoint parameter sets), and a set of three special variables to control the execution. 
Definition 3 (Memory State) A memory state $s$ is composed of:

- two total functions $V_{C} \rightarrow V A L$ and $P_{C} \rightarrow V A L \cup\{\perp\}$, where VAL is the set of all values of the different Java types and $\perp \notin V A L$; the former function assigns a value to any attribute and ghost variable of $C$; the latter assigns a value to any parameter of any method of $C$; when the parameter is not used, its value is undefined - denoted $\perp$.

- a boolean variable excp; the predicate s(excp) indicates that an exception has been thrown,

- a variable $c M \in M_{C}$, indicating the name of the method currently performed,

- a variable sH, that is a natural number that represents the height of the execution stack.

This definition simplifies memory models for object oriented languages [22, 29]. The Java memory also contains an execution stack [20]. As in [21], we do not explicitly use the execution stack, but we observe it with the three special variables excp, $c M$ and $s H$. Given a state $s$ and a variable $x$ in $V_{C} \cup P_{C} \cup\{\operatorname{excp}, c M, s H\}, s(x)$ denotes the value of $x$ in the state $s$, when it is defined. We denote by STATE the set of memory states of a class $C$.

Let $E$ be a Java/JML expression. We consider an evaluation function, written $\operatorname{eval}(E, s)$, that returns the value of $E$ in the state $s$. We suppose that expressions are side-effect free and do not contain method calls. They are denoted $E, E_{i}$. JML predicates are boolean expressions defined over attributes, ghost variables and values of their types. Some predicates, for example in the constraint or ensures clauses, are pre-/post-predicates using the values of variables in the previous state by the \old notation. Let $P$ be a JML predicate and $s, s^{\prime}$ be two memory states. If $P$ does not contain the keyword $\backslash$ old, $s \models P$ denotes that $\operatorname{eval}(E, s)=$ true. Otherwise, $\left(s, s^{\prime}\right) \models P$ denotes that the evaluation of $P$ w.r.t. the states $s$ and $s^{\prime}$ is true. The subterms $t$ of $P$ appearing as $\backslash$ old (t) in $P$ are evaluated in the state $s$, and the subterms $t^{\prime}$ that are not included in the keyword \old are evaluated in the state $s^{\prime}$.

Intuitively, we define the semantics of a Java statement $T$ as the execution $s \llbracket T \rrbracket$ that is generated by the execution of $T$ from the memory state $s$ of STATE. An execution is a sequence of memory states, i.e. an element of $S T A T E^{+\omega}$.

Definition 4 (Java Subset Semantics) Let $T$ be a Java statement and $s \in$ STATE a memory state without exception ( $\neg\left(\right.$ excp)). The execution $s \llbracket T \rrbracket$ is defined in Fig. 2, where $f_{\text {as }}:$ STATE $\rightarrow$ STATE is the state transformer of the atomic statement as.

Each equality in this definition must be understood as follows:

1. The execution for an atomic statement as is the output state resulting from as. 
2. The first state $s_{i n}$ is a pre-state that contains the value of every parameter of $m$. In this state, the current method is $m$ and the stack height is incremented. If $s_{i n}$ does not satisfy the precondition $P_{m}$ of method $m$, the execution the execution raises an exception. Otherwise, the state $s_{i n}$ is followed by the sequence of states resulting from the execution of the body of $m$. When this execution is finite, it ends with a last state $s_{\text {exit }}$ whose parameters, $c M$ and $s H$, are equal to their values in the first state $s$ and whose other values are those of the last state of the body execution.

3. The execution for a sequence of $T_{1}$ and $T_{2}$ is the concatenation of the executions of $T_{1}$ and $T_{2}$ if $T_{1}$ terminates without raising an exception. Otherwise, it is the execution of $T_{1}$.

4. The execution for the conditional statement is the execution of $T_{1}$ if $s$ satisfies $P$ and the execution of $T_{2}$ otherwise.

5. Defining the execution semantics of the iterative while statement is a difficult point. For any predicate $P$ and statement $T$ it is expected that this execution is empty if $s$ does not satisfy $P$, is the execution of $T$ if $T$ does not terminate or raises an exception, and is otherwise the concatenation of a first execution of $T$ and the execution of the same iterative statement from the last state of this first execution of $T$. To simplify our semantics we define never an empty execution in such a way the function last is always defined. The semantics of while (false) $\{T\}$ is defined by $s$. This stuttering has no effect on the visibles states. Last, note that this is an expression of the syntactic statement skip. This is the intended meaning of the fifth equality in Fig. 2. The trouble is that this "definition" of $(\lambda s . s \llbracket$ while $(P)\{T\} \rrbracket)$ is circular. The question remains whether this equation admits a solution and, if it admits more than one, which one should be retained as the right definition. A basic answer is to define this solution as a fixpoint over an adequate lattice. Consider the set $\llbracket T \rrbracket$ of executions starting from any memory state in STATE. This set is related to the unique execution $s \llbracket T \rrbracket$ after a given state $s$ by $\llbracket T \rrbracket=\{s . \sigma \mid s \in S T A T E \wedge \sigma \in s \llbracket T \rrbracket\}$. \while $(P)\{T\} \rrbracket$ could be defined in the sequence set lattice from Prop. 2 as the least fixpoint of the endofunction $W$ defined by $W=\left(\lambda X .\left\{\sigma \in S_{T A T E}^{+\omega} \mid \operatorname{len}(\sigma)=1 \wedge \sigma(0) \not \models P\right\} \cup\left\{\sigma \in \operatorname{STATE}^{+\omega} \mid \sigma \in \llbracket T \rrbracket \wedge \sigma(0) \models\right.\right.$ $P \wedge \operatorname{last}(\sigma)($ excp $)\} \cup\left\{\sigma \in S T A T E^{+\omega} \mid \sigma \in \llbracket T \rrbracket \wedge \sigma(0) \models P \wedge \neg \operatorname{last}(\sigma)(\right.$ excp $\left.\left.)\right\}{ }^{\wedge} X\right)$ with the convention that $\omega($ excp $)=$ false. On the one hand, this function is monotone. On the other hand, a proof by induction on the statement language shows that the execution set $W(X)$ contains exactly one execution starting from any given state, for any execution set $X$. Then $s \llbracket$ while $(P)\{T\} \rrbracket$ is defined as the execution starting with $s$ in the least fixpoint of $W$.

6. The execution for the $\operatorname{try}\left\{T_{1}\right\} \operatorname{cat} \operatorname{ch}\left\{T_{2}\right\}$ statement is the execution of $T_{1}$ if $T_{1}$ either does not 
terminate or terminates without raising an exception. Otherwise, when $T_{1}$ terminates, the raised exception is removed and the execution continues with the execution of $T_{2}$.

7. The execution for the $\operatorname{try}\left\{T_{1}\right\}$ finally $\left\{T_{2}\right\}$ statement is the execution of $T_{1}$ if $T_{1}$ does not terminate. Otherwise, when $T_{1}$ terminates either normally or by throwing an exception, the raised exception is caught if necessary and the execution continues with the execution of $T_{2}$.

8. The throw statement assigns the special variable excp to true. If the throw statement is in the $T_{1}$ part of a $\operatorname{try}\left\{T_{1}\right\} \operatorname{catch}\left\{T_{2}\right\}$ statement, the execution continues with the execution of $T_{2}$ as it is specified by its semantics. Otherwise, the execution stops.

\subsection{Class Semantics}

As explained in Sect. 1, we aim to verify that a class $C$ satisfies a liveness property. This satisfaction obviously depends on the context of use of that class. Here, we focus on the life cycle of a single object of type $C$, after its construction. We assume the encapsulation hypothesis, i.e. that the class attributes can be modified only by the invocation of class methods. Consequently, the class use only depends upon the manner invoking the class methods. The class executions result from the activation of the constructor followed by a finite or infinite sequence of method calls that respect the contract - each of them protected by an exception recuperation statement. This class semantics $\Sigma_{C}^{+\omega}$ is defined in this section.

A method execution at toplevel is a (maximal) execution of a method $m$ that starts from any state where the execution stack is empty and the exception flag is down. The set of executions of a class $C$ at toplevel is denoted $\llbracket C \rrbracket$ and defined by

$$
\begin{aligned}
& \llbracket C \rrbracket={ }_{\operatorname{def}}\left\{\quad s . s \llbracket \operatorname{try}\left\{m\left(v\left(p_{1}\right), \ldots, v\left(p_{n}\right)\right)\right\} \operatorname{catch}\{\} \rrbracket \mid m \in M_{C} \wedge\right. \\
& \left.v \in \text { paramList }_{m} \rightarrow V A L \wedge s \in \text { STATE } \wedge \neg s(\operatorname{excp}) \wedge s(s H)=0 \wedge s \models P_{m}\right\}
\end{aligned}
$$

where paramList $_{m}=\left\{p_{1}, \ldots, p_{n}\right\}$.

Let $C$ be an annotated class, STATE its set of states, $\llbracket \rrbracket \in S T A T E^{+\omega}$ its execution semantics and $S_{0} \subseteq S T A T E$ the set of initial states resulting from the constructor $i_{C}$ of $C$. The set $f(C)$ of blocking (or final) states for the class $C$ is defined by $f(C)=S T A T E \backslash\{\sigma(0) \mid \sigma \in \llbracket C \rrbracket\}$. With these notations, the maximal execution semantics of an annotated class can be defined thanks to the following endofunction.

Proposition 3 In the complete lattice $\left\langle 2^{S T A T E^{+\omega}}, \sqsubseteq, \sqcup, \sqcap\right\rangle$, the endofunction $F$ defined by $F(X)=$ $f(C) \cup\left(\llbracket C \rrbracket^{\frown} X\right)$ is monotone. 
Proof Notations are the same as in Prop. 2, except for $S$ replaced here with the set STATE of memory states. By separating finite and infinite sequences, one has $F(X)^{+}=f(C) \cup\left(\llbracket C \rrbracket^{+\frown} X^{+}\right)$and $F(X)^{\omega}=$ $\left(\llbracket C \rrbracket^{+} X^{\omega}\right) \cup \llbracket C \rrbracket^{\omega} . X \sqsubseteq Y$ implies that $F(X)^{+} \subseteq F(Y)^{+}$and $F(X)^{\omega} \supseteq F(Y)^{\omega}$, i.e. $F(X) \sqsubseteq F(Y)$.

When $\llbracket C \rrbracket$ is a transition relation, i.e. when it is a set of executions of length 2 , this proposition is a corollary of Th. 13 from [10], proved by fusion of fixpoints on the two lattices of nonempty finite and infinite executions. The present result is more general, since $\llbracket C \rrbracket$ may contain finite executions of any length, and even infinite executions. A consequence is that a proof by fusion is no more possible.

By Prop. 1 and 3, $F$ admits a leastfixpoint, denoted lfp $(F)$.

Definition 5 (Class Semantics) The restriction of lfp $(F)$ to executions starting from the states resulting from the constructor is called the class semantics and is denoted $\Sigma_{C}^{+\omega}={ }_{\operatorname{def}} l f p(F) \cap\left(S_{0} \frown S T A T E^{+\omega}\right)$.

\subsection{Visible States}

The semantics of the JML invariant and constraint clauses is based on the notion of "visible" states. This section formalises this notion and its semantics. Under the hypotheses of Sect. 4.1, the original definition of visible states, given in the JML reference manual [18], is restricted to three cases, as follows. A visible state is a state that occurs at one of these moments in a program's execution: at the first state of the execution, just after the end of a constructor invocation that has created the executed object; at the beginning or end of a (non-static non-finalizer) method invocation; outside of the execution of any constructor, finalizer, or method when the execution stack is empty.

Let us first formalise the notions of pre- and post-states for a method $m$ as follows.

Definition 6 (Pre- and Post-States) Let $C$ be a class, $\sigma \in \Sigma_{C}^{+\omega}$ an execution, $m$ a method of class $C$ and $0 \leq i<\operatorname{len}(\sigma)$. For $i>0$, the $i^{\text {th }}$ state $\sigma(i)$ of $\sigma$ is a pre-state of $m$, denoted prestate $(\sigma, i, m)$, if $\sigma(i)(c M)=m$ and $\sigma(i)(s H)=\sigma(i-1)(s H)+1$. The $i^{\text {th }}$ state of $\sigma$ is a post-state of $m$, denoted poststate $(\sigma, i, m)$, if $\sigma(i)(c M)=m$ and $\sigma(i)(s H)=\sigma(i+1)(s H)+1$.

With this definition, for any execution of class $C$, we formalise - in conformity with [18] - what a visible state is.

Definition 7 (Visible States) Given an execution $\sigma \in \Sigma_{C}^{+\omega}$, the $i^{\text {th }}$ state $\sigma(i)$ of $\sigma$ is a visible state, denoted visible $(\sigma, i)$, iff $i=0, \sigma(i)(s H)=0$ or there is a method $m \in M_{C}$ in $C$ s.t. prestate $(\sigma, i, m)$ or poststate $(\sigma, i, m)$.

It is now possible to abstract any execution by keeping only its visible states. The following definition of this abstraction is based on an auxiliary partial function $n v: \mathbb{N} \times \Sigma_{C}^{+\omega} \rightarrow \mathbb{N}$, such that $n v(i, \sigma)$ is 
the position of the $i+1$-th visible state in $\sigma$, when it exists. Let $\min (S)$ denote the minimum of any subset $S$ of $\mathbb{N} . n v$ is inductively defined by $n v(0, \sigma)=\min (\{j \mid 0 \leq j<\operatorname{len}(\sigma) \wedge \operatorname{visible}(\sigma, j)\})$ and $n v(i, \sigma)=\min (\{j \mid n v(i-1, \sigma)<j<\operatorname{len}(\sigma) \wedge \operatorname{visible}(\sigma, j)\})$ for $i>0$.

Definition 8 (Visible State Abstraction) The visible state abstraction of a class $C$, denoted vsa ${ }_{C}$, is the endofunction of $\Sigma_{C}^{+\omega}$ defined by $v s a_{C}(\sigma)(i)=\sigma(n v(i, \sigma))$ for any $\sigma$ in $\Sigma_{C}^{+\omega}$ and any $0 \leq i<\operatorname{len}(\sigma)$.

\subsection{Class in Isolation Semantics}

The semantics of a class in isolation is defined as the set of abstractions to visible states of complete (maximal) class executions. Following [21], this execution semantics is called the class in isolation semantics. It is defined as follows:

Definition 9 (Class In Isolation Semantics) The class in isolation semantics of a class $C$ is defined by $\Sigma_{C}=_{\text {def }}\left\{v s a_{C}(\sigma) \mid \sigma \in \Sigma_{C}^{+\omega}\right\}$.

\subsection{Annotated Class Consistency}

To express temporal properties by JML annotations, we need an execution semantics of JML annotations. To our knowledge, JML semantics has been given in terms of wp-calculus (see for example [22]), but never in terms of properties of the executions. In this section, we give an execution semantics of JML annotations defining their consistency with the set of executions $\Sigma_{C}$ of the class in isolation.

In an annotated class, there are three canonical kinds of annotations: invariant, constraint and behavior. Their semantics are given by Def. 11 w.r.t. the definition in [18]. In Def. 11, we use the predicate $m p(\sigma, j, m, i)$ that is true if $\sigma(j)$ is the matching post-state of the pre-state $\sigma(i)$ (Def. 10).

Definition 10 (Matching Post-State of a State for a Method in an Execution) The $j^{\text {th }}$ state of $\sigma \in \Sigma_{C}^{+\omega}$ is the matching post-state of the $i^{\text {th }}$ state of $\sigma$ for method $m$, denoted $m p(\sigma, j, m, i)$, if

$$
\text { poststate }(\sigma, j, m) \wedge \sigma(j)(s H)=\sigma(i)(s H) \wedge \forall k .(i<k<j \Rightarrow \sigma(k)(s H) \geq \sigma(i)(s H)) .
$$

Definition 11 (Consistency) Let $C$ be an annotated class. We define that an execution $\sigma$ of $\Sigma_{C}$ satisfies a JML annotation $\mathcal{A}$ of the class $C$, denoted $\sigma: \mathcal{A}$, according to the formulae in Fig. 3 .

This definition must be understood as follows:

- Invariant: The invariant must be satisfied by each visible state (see (1) in Fig. 3). 
- Constraint: For the body of each method included in the for clause, the constraint must hold between two consecutive visible states that arise during the execution of the method, i.e., all visible states between the pre-state and the matching post-state of the method (see (2) in Fig. 3).

- Behavior method specification: This JML specification is interpreted over an execution as follows. If the predicate $P_{m}$ of the requires clause is satisfied by the pre-state of the method $m$, that implies:

- If $D_{m}$ does not hold $\left(\neg D_{m}\right)$, then the method must terminate, i.e., it must have a poststate. Moreover, if it is a normal termination $(\sigma(j)($ excp $))$, the predicate $Q_{m}$ of the ensures clause must be satisfied between the pre-state and the post-state, and the predicate $R_{m}$ of the signals clause must be satisfied otherwise (see the case $\neg D_{m}$ in (3)).

- If $D_{m}$ holds and the method terminates, then the pre-state and its matching post-state satisfy the same condition postcontract $(\sigma, j, m, i)$ as in the previous case (see the case $D_{m}$ in (3)).

\section{$5 \quad$ Liveness Properties}

Liveness properties extend the notion of program termination by stipulating that a program must eventually reach some given states. This section deals with the expression and verification of liveness properties on a class $C$.

\subsection{Liveness Operator}

The liveness properties under consideration are those expressible by the Loop operator defined in this section. For any state predicate $Q$, the temporal formula $\operatorname{Loop}(Q)$ corresponds to the linear-time temporal logic (LTL) property GF $\neg Q$ for infinite sequences of states. It is also satisfied by finite sequences of states ending in a state where $Q$ does not hold. Its semantics is based on the notion of visible states in JML. It is defined on finite and infinite executions as follows:

Definition 12 (Loop Operator) Let $Q$ be a predicate. The execution $\sigma \in \Sigma_{C}$ satisfies the liveness operator $\operatorname{Loop}(Q)$, written $\sigma \models \operatorname{Loop}(Q)$, if

$$
\forall i .(0 \leq i<\operatorname{len}(\sigma) \Rightarrow \exists j .(i \leq j<\operatorname{len}(\sigma) \wedge \sigma(j) \models \neg Q)) .
$$

This satisfaction relation is lifted up to sets of executions with the semantics that every execution in the set satisfies the formula. 


\subsection{Class Liveness}

In object-oriented programming, defining and checking the satisfaction of a liveness property on a whole program - composed of many classes - may be an heavy task. As a first step, this section presents the semantics of a liveness property attached to a single Java class.

A liveness property $\operatorname{Loop}(Q)$ declared in a class $C$ must hold for every object $o$ of type $C$. For the sake of simplicity, $C$ is assumed to have no static attribute. Thus $Q$ is a JML predicate with variables among the (non-static) attributes of $C$. The satisfaction of $\operatorname{Loop}(Q)$ on an execution of $\Sigma_{C}$ intuitively means that if, during the execution, any instance of the class $C$ is in a state satisfying $Q$, then it is always possible to reach a state satisfying $\neg Q$ by invoking methods of $C$ on this instance. In other words, $C$ satisfies the liveness property $\operatorname{Loop}(Q)$ if $\Sigma_{C}=\operatorname{Loop}(Q)$.

\subsection{Proving Liveness}

Along the line of Floyd's total correctness proof method, we plan to prove liveness with the help of a variant function that assigns a value to each program state. That value should decrease at each program step, according to a well-founded ordering. In the deterministic case, it is sufficient [11] to consider variants taking their values in $\mathbb{N}$, totally ordered with $<$.

In the present case, some program steps are calls to methods of a class $C$. It is obvious that a call to a side-effect free method of $C$ cannot change the value of any variant. Thus, the variant of a liveness property will be required to decrease strictly for a subset of methods with side effects. Consequently, when assigning a liveness property to a Java class, the user is asked to specify a variant $V$ and a set $M$ of progress methods. This extension of the Loop operator with $V$ and $M$, attached to a class $C$, is denoted $\operatorname{Loop}_{C}(Q, V, M)$.

In order to verify $\Sigma_{C} \models \operatorname{Loop}_{C}(Q, V, M)$, we need to assume progress of the environment, i.e., that the environment invokes the methods of the subset $M$.

Definition 13 (Progress Hypothesis) For any set of methods $M$, an execution $\sigma \in \Sigma_{C}$ satisfies the progress hypothesis, written $\sigma \models P H(M)$, if

$$
\forall i .\left(0 \leq i<\operatorname{len}(\sigma) \Rightarrow \exists j .\left(i \leq j<\operatorname{len}(\sigma) \wedge \bigvee_{m \in M} \operatorname{prestate}(\sigma, j, m)\right)\right)
$$

This satisfaction relation extends to sets of executions in a standard way. The semantics of $\operatorname{Loop}_{C}(Q, V, M)$ is given by the following definition, where $1_{M}$ is the characteristic function of set $M$, whose value $1_{M}(m)$ at $m$ is 1 if $m \in M, 0$ otherwise: 
Definition 14 (Liveness Clause) Let $C=\left(V_{C}, I_{C}, C_{C}, M_{C}\right)$ be an annotated class, $Q$ a predicate on the attributes of $C, V: V_{C} \rightarrow \mathbb{N}$ a variant function, and $M \subseteq M_{C}$ a set of methods of $C$. An execution $\sigma \in \Sigma_{C}$ satisfies the liveness clause $\operatorname{Loop}_{C}(Q, V, M)$, written $\sigma \models \operatorname{Loop}_{C}(Q, V, M)$, if

$$
\sigma \models P H(M) \Rightarrow \forall i .\left((0 \leq i<\operatorname{len}(\sigma)-1) \Rightarrow\left(\sigma(i) \models Q \Rightarrow \bigwedge_{m \in M_{C}} V(\sigma(i))-V(\sigma(i+1)) \geq 1_{M}(m)\right)\right) .
$$

The variant-based liveness proof method is summarised in the following proposition:

Proposition 4 For any execution $\sigma \in \Sigma_{C}$ satisfying the progress hypothesis $P H(M)$, if $\sigma \models \operatorname{Loop}_{C}(Q, V, M)$ then $\sigma=\operatorname{Loop}(Q)$.

\subsection{Approximation with JML Annotations}

This section shows how to use existing JML tools for verifying liveness properties on a class in isolation. The idea is to replace the liveness clause with standard JML annotations, whose satisfaction is sufficient to establish $\Sigma_{C} \models \operatorname{Loop}_{C}(Q, V, M)$.

Verification of the $\operatorname{Loop}_{C}(Q, V, M)$ property is quite similar to a termination proof. As long as $Q$ holds, it must be possible to invoke a method of $M$, and methods in $M$ must decrease the variant $V$. Here we propose proof obligations - inspired from [9] - expressed as JML annotations. These proof obligations guarantee the satisfaction of the $\operatorname{Loop}_{C}(Q, V, M)$ property by the executions of the class $C$ in isolation.

Let $\mathcal{A}_{1-5}$ be the following set of JML annotations:

$$
\begin{aligned}
& \text { invariant } V>=0 ; \\
& \text { constraint } Q==>V<\operatorname{lold}(V) \text { for } M ; \\
& \text { constraint } Q==>V<=\operatorname{lold}(V) ; \\
& \text { invariant } Q==>\bigvee_{m \in M} P_{m} ; \\
& \text { invariant } Q==>\bigwedge_{m \in M_{C}}\left(P_{m}==>! D_{m}\right) ;
\end{aligned}
$$

Remember that JML invariants have to hold on all visible states, and JML constraints have to hold between any two successive visible states [18]. These annotations $\mathcal{A}_{1-5}$ relate to $Q, V, M$, and a class $C$ and its methods as follows:

$\mathcal{A}_{1}$ The variant $V$ is actually greater than zero, it is a function returning a natural number. 
$\mathcal{A}_{2}$ As long as $Q$ holds, the variant $V$ must decrease when a method in $M$ is executed.

$\mathcal{A}_{3}$ As long as $Q$ holds, the variant $V$ must not increase when a method of $C$ is executed.

$\mathcal{A}_{4}$ As long as $Q$ holds, there should always be a method in $M$ that may be called, i.e., whose precondition $P_{m}$ (in the clause requires $P_{m}$ ) holds. This ensures the deadlock-freeness of the system.

$\mathcal{A}_{5}$ As long as $Q$ holds, all callable methods must not diverge, according to the clause diverges $D_{m}$. This ensures the non-divergence of the system.

In the rest of the paper, $\sigma: \mathcal{A}_{1-5}$ denotes $\sigma: \mathcal{A}_{1} \wedge \ldots \wedge \sigma: \mathcal{A}_{5}$.

Theorem 1 Let $\sigma \in \Sigma_{C}$ be an execution. If $\sigma: \mathcal{A}_{1-5}$ then $\sigma \models \operatorname{Loop}_{C}(Q, V, M)$.

Proof 1 There are two cases:

1. If $\sigma \in \Sigma_{C}$ is a finite execution, the definitions in Sect. 4 imply that last $(\sigma) \not \models P_{m}$ for any method $m$ in $M_{C}$, and that last $(\sigma)$ is the prestate of no method. That falsifies $P H(M)$ for any $M \subseteq M_{C}$ when $i=\operatorname{len}(\sigma)-1$. Thus $\sigma \models \operatorname{Loop}_{C}(Q, V, M)$.

2. If $\sigma$ is an infinite execution, the proof is by contradiction. Suppose there exists $\sigma \in \Sigma_{C}$ such that $\sigma \not \models \operatorname{Loop}_{C}(Q, V, M)$. By Def. 13 and 14,

$$
\sigma \models P H(M)
$$

and there are some $i, 0 \leq i<\operatorname{len}(\sigma)-1$ and some method $m \in M$, s.t. $\sigma(i) \models Q$ and

$$
V(\sigma(i))-V(\sigma(i+1))<1_{M}(m)
$$

Since $\sigma \in \Sigma_{C}$, by the progress hypothesis (Def. 13), we have:

$$
\forall k \geq 0 . \exists k_{2} \geq k . \exists m \in M . p r e s t a t e\left(\sigma, k_{2}, m\right)
$$

The above property being true for each index $k \geq 0$, it is also the case for each index $k \geq i$ :

$$
\forall k \geq i . \exists k_{2} \geq k . \exists m \in M . p r e s t a t e\left(\sigma, k_{2}, m\right) .
$$


(Def. 6), we derive:

$$
\forall k \geq 0 . \forall m \in M_{C} . \text { prestate }(\sigma, k, m) \Rightarrow \sigma(k) \models P_{m} .
$$

On the one hand, from (3) and (4), we obtain:

$$
\forall k \geq i . \exists k_{2} \geq k . \exists m \in M . \text { prestate }\left(\sigma, k_{2}, m\right) \wedge \sigma\left(k_{2}\right) \models P_{m}
$$

On the other hand, from (2) and $\left(\mathcal{A}_{5}\right)$, we have:

$$
\forall m \in M . \sigma\left(k_{2}\right) \models P_{m} \Rightarrow \sigma\left(k_{2}\right) \not \models D_{m} .
$$

Then, from (5) and (6), we obtain:

$$
\forall k \geq i . \exists k_{2} \geq k . \exists m \in M . \text { prestate }\left(\sigma, k_{2}, m\right) \wedge \sigma\left(k_{2}\right) \models P_{m} \wedge \sigma\left(k_{2}\right) \not \models D_{m} .
$$

By Def. 11 (Fig. 3), when using default values [18] of all but $D_{m}$ of the behavior clause on $\sigma$ above, item (3) results in:

$$
\forall k \geq i . \exists k_{2} \geq k . \exists m \in M . \text { prestate }\left(\sigma, k_{2}, m\right) \wedge \exists k_{3} \geq k_{2} . m p\left(\sigma, k_{3}, m, k_{2}\right) .
$$

By $\left(\mathcal{A}_{2}\right),(8)$ and transitivity of history constraints (Def. 11, item (2) in Fig. 3), we obtain:

$$
\begin{aligned}
& \forall k \geq i . \exists k_{2} \geq k . \exists m \in M . \\
& \operatorname{prestate}\left(\sigma, k_{2}, m\right) \wedge \exists k_{3} \geq k_{2} . m p\left(\sigma, k_{3}, m, k_{2}\right) \wedge\left\langle\sigma\left(k_{2}\right), \sigma\left(k_{3}\right)\right\rangle \models V<\backslash \operatorname{old}(V) .
\end{aligned}
$$

By a similar reasoning, we also obtain, from $\left(\mathcal{A}_{3}\right)$ :

$$
\begin{aligned}
& \forall k \geq i . \exists m \in M_{C} . \\
& \operatorname{prestate}(\sigma, k, m) \Rightarrow \exists j \geq k . m p(\sigma, j, m, k) \wedge\langle\sigma(k), \sigma(j)\rangle \models V \leq \backslash \operatorname{old}(V) .
\end{aligned}
$$

Consequently, from (9) and (10) one deduces that the variant $V$ decreases infinitely during the execution. And so, $\mathcal{A}_{1}$ cannot be established. A contradiction.

In JML side-effect free methods can be identified syntaxically thanks to the keyword pure. Let Pure $C$ 
be the set of pure methods of the class $C$. Let $\mathcal{P} \mathcal{M}_{C}=M_{C} \backslash$ Pure $_{C}$ denote the set of so-called progress methods of the class $C$, i.e. with a side effect. An interesting property is obtained when $M=\mathcal{P} \mathcal{M}_{C}$. In this particular case, the progress hypothesis $P H(M)$ is not only sufficient but also necessary.

Proposition 5 Let $\sigma \in \Sigma_{C}$ be an execution. If $\sigma \models \operatorname{Loop}_{C}\left(Q, V, \mathcal{P} \mathcal{M}_{C}\right)$ and $C: \mathcal{A}_{1-5}$ then $\sigma \models$ $\operatorname{PH}\left(\mathcal{P} \mathcal{M}_{C}\right)$.

\section{Liveness Temporal Patterns}

In [15], we have presented a way to verify liveness properties expressed with the Loop ${ }_{C}$ operator. This section presents a practical context of Java/JML verification where this verification method is applied.

Along the line of helping Java programmers in writing formal specifications, Trentelman and Huisman [28] proposed a temporal extension of JML inspired by the pragmatic work of the SanTos Specification Pattern Project [12]. We refer to this temporal extension of JML as JTPL, for Temporal Pattern Language, prefixed by a ' $\mathrm{J}$ ' to denote its adaptation to Java. The semantics of temporal formulae in JTPL and translation rules into JML annotations are detailed in [28] for safety properties and in [1] for liveness properties. This section defines a verification technique for liveness properties expressible in JTPL, a problem left open by Trentelman and Huisman [28]. This verification is performed by translating these properties into the $\operatorname{Loop}_{C}$ operator.

\subsection{Language Overview}

JTPL provides the user with patterns to express common temporal requirements of Java classes. Moreover, the language deals with normal and abnormal method terminations. JTPL is based on the notion of trace property which is either always $P$, eventually $P$, or the conjunction or disjunction of two trace properties. always $P$ is true on an execution $\sigma$ if $P$ holds on every state of $\sigma$. eventually $P$ is true on an execution $\sigma$ if $P$ holds on at least one state of $\sigma$.

It is often useful to reduce the scope of a trace property, i.e. specifying it only for subparts of an execution. This is made possible by the notion of event. An event can be: (i) $m$ called, denoting that the method $m$ has been invoked; (ii) $m$ normal, denoting that the method $m$ has terminated normally, i.e., without throwing any exception; (iii) $m$ exceptional, denoting that the method $m$ has terminated by throwing an exception; or (iv) $m$ terminates, denoting that the method $m$ has terminated either normally or by throwing an exception.

Now, a temporal property in JTPL is inductively defined as follows: let $E$ be a disjunction of events, 
$C$ a trace property and $T$ a temporal property. A temporal property can be either: (a) after $E T$, which is true on an execution $\sigma$ if the suffix of $\sigma$ starting after each occurrence of an event in $E$ satisfies the temporal formula $T$; (b) before $E C$, which is true on an execution $\sigma$ if the prefix of $\sigma$ ending with each occurrence of an event in $E$ satisfies the trace property $C$; (c) $C$ until $E$, which is true on an execution $\sigma$ if an event in $E$ occurs and if the trace property $C$ is satisfied on the segment of $\sigma$ ending with an event in $E$; (d) $C$ unless $E$, which is true on an execution $\sigma$ if an event in $E$ occurs and the trace property $C$ is satisfied on the segment of $\sigma$ ending with an event in $E$, or the trace property $C$ is satisfied on the whole execution $\sigma$ and $E$ never happens; or (e) between $E E^{\prime} C$, which is true on an execution $\sigma$ if the temporal formula after $E\left(C\right.$ until $\left.E^{\prime}\right)$ holds on $\sigma$, or (f) a trace property $C$.

\subsubsection{Safety and Liveness Characterisation}

The properties described by this extension of JML are either safety properties or liveness properties. The following proposition makes it possible to distinguish them syntactically:

Proposition 6 (Characterisation of Safety and Liveness Properties) The properties containing only the keywords after, before, unless and always are safety properties. The properties containing the keyword eventually iff they contain the keyword before also are safety properties. The other properties are liveness properties.

For liveness properties, the verification is based on the decrease of a well-founded variant given by the user. Therefore, we propose to extend the syntax of liveness formulae with the following clause:

under [invariant $<$ JMLProp $>$ ] variant $<$ JMLExpr $>$ [for $<$ Methods $>$ ]

In the above clause, $<$ JMLProp $>$ is a JML predicate which is an optional local invariant - like a loop invariant - that can help the proof, <JMLExpr $>$ is the variant expression (its type is a natural number), and $<$ Methods $>$ is a list of Java method names.

\subsubsection{Back to the Example}

Using JTPL formulae, one can express the following properties on the Buffer example (Fig. 1 Sect. 2):

1. After the invocation of storeData (after storeData called), the variable customized is always true, expressed in JTPL as follows:

after storeData called always customized; 
2. After starting a transaction, i.e., after normal termination of the method begin (after begin normal), a state where trDepth is false must eventually be reached.

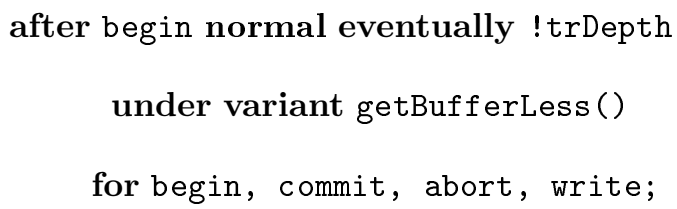

Property $S$ is a safety property and property $L$ is a liveness property. Notice that in $(L)$, the event is begin normal and not begin called since a buffer transaction starts only when the method begin terminates normally. Notice also that since $(L)$ is a liveness property, the user has to give a variant and a set of progress methods with the JTPL clause under variant ... for. Here, the variant corresponds to the free space in the Buffer, and the for clause contains a list of methods that can potentially modify the value of the variant. So, storeData is not in the list.

\subsection{Embedding Liveness Properties into the Loop Clause}

This section presents a translation of a JTPL liveness property into a Loop ${ }_{C}$ clause completed with other JML annotations. Firstly, we present the translation for the basic after $E$ eventually $P$ liveness property. Then, we generalise to the other JTPL liveness properties.

Let us consider a temporal formula of the form:

after $E$ eventually $P$ under variant $V$ for $M$.

To translate liveness JTPL properties, like (11), into a Loop ${ }_{C}$ clause, one needs to observe whether a particular event has already occurred or whether a state satisfying a predicate has already been reached. For that, we define a witness primitive, denoted $\operatorname{JML}\left(X_{1}, X_{2}\right)$, where $X_{1}$ and $X_{2}$ are either JML predicates or JTPL events. Intuitively, given an execution $\sigma, \operatorname{JML}\left(X_{1}, X_{2}\right)$ is satisfied on all states of $\sigma$ between the states satisfying $X_{1}$ and $X_{2}$.

Definition 15 (witness Primitive) Let $\sigma$ be an execution and $i$ a natural number between 0 and len $(\sigma)-1 . \quad$ A state $\sigma(i)$ satisfies $\operatorname{JML}\left(X_{1}, X_{2}\right)$ iff $\exists j .\left(0 \leq j<i \wedge \sigma(j) \models X_{1} \wedge \forall k .(j<k<\right.$ $\left.\left.i \Rightarrow \sigma(k) \not \models X_{2}\right)\right)$.

The witness primitives are expressed by JML ghost variables that are assigned w.r.t. events occurring in the formula. The general rules can be easily derived from the following examples: 
Example 1 (Ghost Variables Generation for $S$ ) The ghost variable witness_S corresponds to the event storeData called of $S$. It is initially declared with the value false (see annotation $S_{a}$ in Fig. 5) and it is set to true when the method storeData is called (see annotation $S_{b}$ ). So, in each state after the event storeData called, the value of the ghost variable witness_S is true, i.e., witness_S is true exactly with the scope of the property.

Example 2 (Ghost Variables Generation for $L$ ) The ghost variable witness_L, corresponding to the event begin normal of the temporal property $L$ is also declared with the value false (annotation $L_{a}$ in Fig. 5). The ghost variable witness_L is assigned using a try $\left\{\operatorname{try}\left\{T_{1}\right\} \operatorname{catch}\left\{T_{2}\right\}\right\}$ finally $\left\{T_{3}\right\}$ statement (see annotation $L_{b}$ ). Notice that in the case of exception, the caught exception is re-thrown. The reader can see that witness_L is set to true only when begin normal occurs. The ghost variable witness_L is set to $\neg$ trDepth again by adding a set statement (annotation $L_{c}$ ) to each method.

Thanks to an adequate witness, one can give a Loop ${ }_{C}$ clause ensuring property (11). Using the semantics of JTPL in [1] and the semantics in Sect. 4, one can show that property (11) holds on the execution $\sigma$ if $\sigma=\operatorname{Loop}_{C}(\operatorname{JML}(E, P), V, M)$.

In a similar way, the other JTPL liveness patterns can be translated into JML annotations (using the Loop $_{C}$ clause) by the rules given in Fig. 4 . For each $\operatorname{Loop}_{C}(Q, V, M)$, the local invariant $J$ is expressed by an invariant clause invariant $Q==>J$. The safety part of the property is also translated into an invariant.

Example 3 (Generation of annotations for $L$ ) The JML translation of $L$ is

$$
\text { Loop } \left._{C} \text { (witness_L, getBufferLess (), \{begin, commit, abort, write }\right\} \text { ). }
$$

The corresponding annotations are displayed in Fig. 5 (see annotations $L_{l o o p}$ ). Notice that, since no method of Buffer diverges, annotation $\mathcal{A}_{5}$ does not appear.

\section{JML Annotation Generator}

The automatic generation of JML annotations for safety properties in [28] and for liveness properties in Sect. 5 has been implemented in a tool, called JAG (for JML Annotation Generator) [13]. The JAG 0.1 release parses a Java file - possibly already JML annotated - with the JML parser included in the Common JML tools and takes a file containing temporal formulae as other input. JAG is freely available from page http://jag.univ-fcomte.fr. 
Translating Temporal Formulae into Intermediate Primitives. The tool reduces each temporal property into one or more intermediate primitives, like the witness primitive, that are semantically equivalent $[28,1]$. These primitives are an internal format which is independent of the JML syntax, allowing an easy extension of the annotation generation to other specification languages, such as Spec

Translating Intermediate Primitives into Standard JML Annotations. Each intermediate Inv primitive representing the safety part of a property is translated into a JML invariant. Each intermediate Loop primitive representing the liveness part of the property is translated into a set of invariants and history constraints that imply the decreasing of the variant and the deadlock-freeness of the system. Each witness is translated into a JML ghost variable. Finally, the tool generates an output file including the original file and enriched with the generated JML annotations. Figure 5 contains the result of the translation of Properties $S$ and $L$.

Example 4 (Invariant Generation for $S$ ) The invariant for $S$ is displayed in Fig. 5 (annotation $S_{c}$ ). It means that when the variable witness_S is true, i.e., after the first occurrence of storeData called, the predicate must be true - the definition of property $S$.

Trace Preservation. The tool is able to keep the trace of the generated annotations, i.e. it is possible, given a generated annotation, to find the original intermediate primitive and the original temporal property.

Experiments. Since the generated output file contains standard JML annotations, it can be used with other JML tools [7] to validate or prove the temporal formulae. For instance, Table 1- where "PO" stands for "Proof Obligation" - summarises the results we have obtained with the JACK tool [8]. All the 277 POs in 4th column have been proved either fully automatically (for 274 POs) or interactively (for remaining 3 POs by enforcing invariants) with the B4free tool as a back-end theorem prover.

TransactionSystem and AtmTransaction are two academic examples. TransactionSystem is adapted from [28] and inspired by the JavaCard transaction mechanism, that ensures that every transaction in a smart card is atomic. AtmTransaction implements a transactional mechanism between a smard card and a terminal. Notice that our theoretical contributions have been applied not only to that academic examples but also to the Demoney system, a Java Card Electronic Purse application we have developed in the framework of an industrial collaboration with Trusted Logic ${ }^{2}$, via the ACI GECCOO project. For this application ${ }^{3}$, we wrote over 500 lines of JML annotations.

\footnotetext{
${ }^{2}$ http://www.trusted-logic.com/

${ }^{3}$ whose demonstrative electronic purse - card specification is available at http://www.doc.ic.ac.uk/ siveroni/secsafe/.
} 
Moreover, we have successfully used the JAG tool for the following purposes:

- Verification of the correctness of the Java code w.r.t. the JML annotations with the proof obligation generators Jack [8] and Krakatoa [22];

- Validation of a JML model with JML-TT [5];

- Formal verification of a JML model with the JML2B method [2];

- Test generation and Runtime Assertion Checking with the test generators Tobias [19], Jartege [24] and JML-TT [4].

Test generation and Runtime Assertion Checking using JAG has been studied on an industrial Java Card application [3].

\section{Conclusion and Future Works}

This paper presents a way to verify liveness properties on Java classes in isolation by generating appropriate JML annotations. This requires that the user specify a variant for the verification of a Loop clause to which liveness properties are reduced. The generated JML annotations are verified (or validated) with any tool handling JML. The JAG tool implements this translation. It has been used for several toy examples and a Java Card Electronic Purse Specification (over 500 lines of JML).

To the best of our knowledge, this is the first attempt to verify liveness properties for potentially infinite-state systems using a translation into JML. We are working on extensions of JAG to other temporal properties. In particular, we currently address the verification of properties expressed by Büchi automata. Assuming that a liveness is established on the class in isolation, another challenge is to provide techniques for verifying that the (single- or multi-threaded) environment effectively satisfies a progress hypothesis.

\section{Acknowledgements}

We would like to thank Pierre Lescanne for his helpful advice, Marieke Huisman for her interesting and helpful comments and suggestions to improve this work, and the anonymous referees for their corrections, comments and advice. 


\section{References}

[1] F. Bellegarde, J. Groslambert, M. Huisman, J. Julliand, and O. Kouchnarenko. Verification of liveness properties with JML. Technical Report RR-5331, INRIA, 2004.

[2] F. Bouquet, F. Dadeau, and J. Groslambert. Checking JML specifications with B machines. ZB'05, volume 3455 of $L N C S$, pages $435-454$. Springer, 2005.

[3] F. Bouquet, F. Dadeau, J. Groslambert, and J. Julliand. Safety property driven test generation from JML specifications. FATES/RV'06, volume 4262 of LNCS, pages 225-239. Springer, 2006.

[4] F. Bouquet, F. Dadeau, and B. Legeard. Automated Boundary Test Generation from JML Specifications. FM'06, volume 4085 of $L N C S$, pages 428-443. Springer, 2006.

[5] F. Bouquet, F. Dadeau, B. Legeard, and M. Utting. JML-Testing-Tools: a symbolic animator for JML specifications using CLP. TACAS'05 Tool session, volume 3440 of LNCS, pages 551-556. Springer, 2005.

[6] C-B. Breunesse, N. Cataño, M. Huisman, and B. Jacobs. Formal methods for smart cards: an experience report. Sci. Comput. Program., 55(1-3):53-80, 2005.

[7] L. Burdy, Y. Cheon, D. Cok, M. Ernst, J. Kiniry, G.T. Leavens, K.R.M. Leino, and E. Poll. An Overview of JML Tools and Applications. FMICS 03, volume 80 of ENTCS, pages 73-89. Elsevier, 2003.

[8] L. Burdy, A. Requet, and J.-L. Lanet. Java Applet Correctness: a Developer-Oriented Approach. FM'03, volume 2805 of $L N C S$, pages 422-439. Springer, 2003.

[9] R.M. Burstall. Program Proving as Hand Simulation with a Little Induction. Information Processing, pages 308-312, 1974.

[10] P. Cousot. Constructive design of a hierarchy of semantics of a transition system by abstract interpretation. Electr. Notes Theor. Comput. Sci., 6, 1997. 25 pages.

[11] E. W. Dijkstra. On weak and strong termination. Selected Writings on Computing: A Personal Perspective, pages 355-357. Springer, 1982.

[12] M. B. Dwyer, G. S. Avrunin, and J. C. Corbett. Patterns in property specifications for finite-state verification. ICSE, pages 411-420, 1999. 
[13] A. Giorgetti and J. Groslambert. JAG: JML Annotation Generation for verifying temporal properties. FASE'2006, Fundamental Approaches to Software Engineering, volume 3922 of LNCS, pages 373-376. Springer, 2006.

[14] A. Giorgetti and J. Groslambert. Un programme annoté en vaut deux. JFLA'07, Journées francophones des langages applicatifs, pages 87-101, Aix-les-Bains, France, January 2007. INRIA.

[15] J. Groslambert, J. Julliand and O. Kouchnarenko. JML-based Verification of Liveness Properties on a Class in Isolation. SAVCBS'06, Specification and Verification of Component-Based Systems,, pages 41-48, Portland, Oregon, USA, November 2006.

[16] B. Jacobs, C. Marché, and N. Rauch. Formal Verification of a Commercial Smart Card Applet with Multiple Tools. AMAST'04, volume 3116 of LNCS, pages 21-22. Springer, 2004.

[17] L. Lamport. Proving the Correctness of Multiprocess Programs. IEEE Transactions on Software Engineering, volume 3(2), pages 125-143, 1977.

[18] G.T. Leavens, E. Poll, C. Clifton, Y. Cheon, C. Ruby, D.R. Cok, and J. Kiniry. JML Reference Manual. Department of Comp. Science, Iowa State University. Available from http://www.jmlspecs.org, 2003.

[19] Y. Ledru, L. du Bousquet, O. Maury, and P. Bontron. Filtering TOBIAS Combinatorial Test Suites. FASE 2004, volume 2984 of LNCS, pages 281-294. Springer, 2004.

[20] T. Lindholm and F. Yellin. The Java Virtual Machine Specification. The Java Series. Addison-Wesley, Reading, MA, USA, 1997.

[21] F. Logozzo. Class Invariants as Abstract Interpretation of Trace Semantics. Computer Languages, Systems and Structures, to appear.

[22] C. Marché, C. Paulin-Mohring, and X. Urbain. The Krakatoa tool for certification of Java/Java Card programs annotated in JML. Journal of Logic and Algebraic Programming, 58(1-2):89-106, 2004 .

[23] B. Meyer. Object-Oriented Software Construction. Prentice Hall, $2^{\text {nd }}$ rev. edition, 1997.

[24] C. Oriat. Jartege: A Tool for Random Generation of Unit Tests for Java Classes. SOQUA 2005, volume 3712 of $L N C S$, pages $242-256$. Springer, 2005.

[25] A. D. Raghavan and G. T. Leavens. Desugaring JML method specifications. Technical Report 00-03d, Iowa State University, Department of Computer Science, July 2003. 
[26] Robby, E. Rodríguez, M. Dwyer, and J. Hatcliff. Checking Strong Specifications Using an Extensible Software Model Checking Framework. TACAS 2004, volume 2988 of LNCS, pages 404-420. Springer, 2004.

[27] A. Tarski. A lattice-theoretical fixpoint theorem and its applications. Pacific Journal of Mathematics, $5(2): 285-309,1955$.

[28] K. Trentelman and M. Huisman. Extending JML Specifications with Temporal Logic. AMAST'02, volume 2422 of $L N C S$, pages 334-348. Springer, 2002.

[29] J. van den Berg, M. Huisman, B. Jacobs, and E. Poll. A Type-Theoretic Memory Model for Verification of Sequential Java Programs. WADT, volume 1827 of $L N C S$, pages 1-21. Springer, 1999. 


\section{List of Figures}

1 JML annotated transaction system. Every opened transaction must eventually be closed . 28

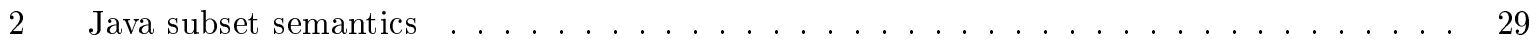

3 Consistency between JML annotations and executions $\ldots \ldots \ldots \ldots$

4 Translation of JTPL liveness patterns using the Loop $_{C}$ clause $\ldots \ldots \ldots \ldots$

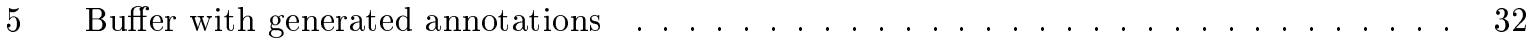




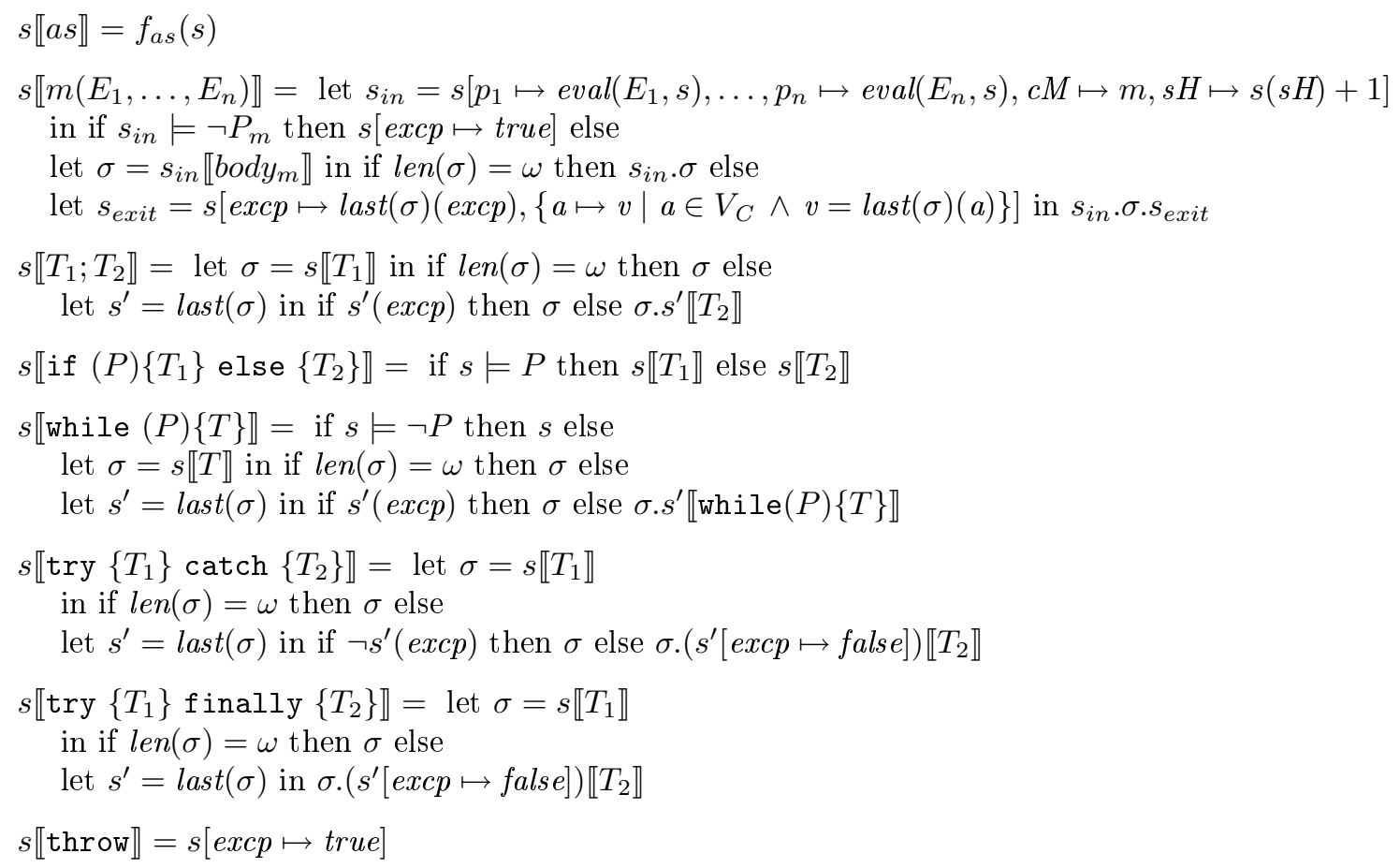

Figure 2: Java subset semantics 
(1) $\sigma$ : invariant $I$ if $\forall i \geq 0 . \sigma(i) \mid=I$.

(2) $\sigma$ : constraint $H$ for $M$ if $\forall i \geq 0 . \forall m \in M .($ prestate $(\sigma, m, i) \Rightarrow \forall j, k .(m p(\sigma, j, m, i) \wedge i<k \leq j \Rightarrow(\sigma(k-1), \sigma(k)) \models H))$.

$\sigma$ : behavior; requires $P_{m}$; diverges $D_{m}$; ensures $Q_{m}$; signals (Exception e) $R_{m}$; if (3) $\forall i \geq 0 .\left(\right.$ prestate $(\sigma, m, i) \wedge \sigma(i) \models P_{m} \Rightarrow$ $\left(\sigma(i)=\neg D_{m} \Rightarrow \exists j>i .(m p(\sigma, j, m, i) \wedge \operatorname{postcontract}(\sigma, j, m, i))\right) \wedge$ $\left.\left(\sigma(i) \mid=D_{m} \Rightarrow \forall j>i .(m p(\sigma, j, m, i) \Rightarrow \operatorname{postcontract}(\sigma, j, m, i))\right)\right)$

where postcontract $(\sigma, j, m, i)=\left(\neg \sigma(j)(\right.$ excp $\left.) \Rightarrow(\sigma(i), \sigma(j)) \models Q_{m}\right) \vee\left(\sigma(j)(\right.$ excp $\left.) \Rightarrow(\sigma(i), \sigma(j)) \models R_{m}\right)$

Figure 3: Consistency between JML annotations and executions 


\begin{tabular}{|c|c|}
\hline Temporal Formula & Translation \\
\hline $\begin{array}{l}\text { eventually } P \text { under invariant } J \\
\text { variant } V \text { for } M\end{array}$ & $\begin{array}{l}\text { Loop }_{C}(\neg J M L(P, \text { false }), V, M) \\
/ / @ \text { invariant } \neg J M L(P, \text { false })==>J ;\end{array}$ \\
\hline $\begin{array}{l}\text { always } P \text { until } E \text { under } \\
\text { invariant } J \text { variant } V \text { for } M\end{array}$ & $\begin{array}{l}\text { Loop }_{C}(\neg \mathrm{JML}(E, \text { false }), V, M) \\
/ / @ \text { invariant } \neg J M L(E, \text { false })=\Rightarrow P \text { \&\& } J\end{array}$ \\
\hline $\begin{array}{l}\text { eventually } P \text { under invariant } J \\
\text { variant } V \text { for } M \text { unless } E\end{array}$ & $\begin{array}{l}\text { Loop } p_{C}(\neg J M L(P, \text { false }), V, M) \\
/ / @ \text { invariant } J M L(E, \text { false })=\Rightarrow \mathrm{JML}(P, \text { false }) \\
/ / \odot \text { invariant } \neg \mathrm{JML}(P, \text { false })=\Rightarrow J ;\end{array}$ \\
\hline $\begin{array}{l}\text { eventually } P \text { until } E \\
\text { under invariant } J \\
\text { variant } V \text { for } M\end{array}$ & $\begin{array}{l}\text { Loop }_{C}(\neg \mathrm{JML}(E, \text { false }), V, M) \\
/ / @ \text { invariant } \mathrm{JML}(E, \text { false })=\Rightarrow \mathrm{JML}(P, \text { false }) \\
/ / \odot \text { invariant } \neg \mathrm{JML}(E, \text { false })==J J\end{array}$ \\
\hline $\begin{array}{l}\text { after } E_{1} \text { always } P \text { until } E_{2} \\
\text { under invariant } J \text { variant } V \text { for } M\end{array}$ & $\begin{array}{l}\text { Loop }_{C}\left(\mathrm{JML}\left(E_{1}, E_{2}\right), V, M\right) \\
/ / \Theta \text { invariant } \operatorname{JML}\left(E_{1}, E_{2}\right)=\Rightarrow P \text { \&\& J; }\end{array}$ \\
\hline $\begin{array}{l}\text { after } E \text { eventually } P \text { under } \\
\text { invariant } J \text { variant } V \text { for } M\end{array}$ & $\begin{array}{l}\text { Loop }_{C}(\mathrm{JML}(E, P), V, M) \\
/ / \Theta \text { invariant } \mathrm{JML}(E, P)=\Rightarrow P \text { \&\& } J\end{array}$ \\
\hline $\begin{array}{l}\text { after } E_{1} \text { eventually } P \text { until } E_{2} \text { under } \\
\text { invariant } J \text { variant } V \text { for } M\end{array}$ & $\begin{array}{l}\operatorname{Loop}_{C}\left(\mathrm{JML}\left(E_{1}, E_{2}\right), V, M\right) \\
/ / @ \text { invariant } \mathrm{JML}\left(E_{2}, E_{1}\right)=\Rightarrow \mathrm{JML}\left(\left(\mathrm{JML}\left(E_{1}, E_{2}\right) \wedge P\right), E_{1}\right) \& \& \quad J ;\end{array}$ \\
\hline $\begin{array}{l}\text { after } E_{1} \text { eventually } P \text { under } \\
\text { invariant } J \\
\text { variant } V \text { for } M \text { unless } E_{2}\end{array}$ & $\begin{array}{l}\text { Loop }_{C}\left(\mathrm{JML}\left(E_{1}, P\right), V, M\right) \\
/ / @ \text { invariant } \mathrm{JML}\left(E_{2}, E_{1}\right)=\Rightarrow \mathrm{JML}\left(\left(\mathrm{JML}\left(E_{1}, E_{2}\right) \wedge P\right), E_{1}\right) \\
/ / @ \text { invariant } \mathrm{JML}\left(E_{1}, P\right)=\Rightarrow J\end{array}$ \\
\hline
\end{tabular}

Figure 4: Translation of JTPL liveness patterns using the Loop ${ }_{C}$ clause 
public class Buffer \{

//@ ghost boolean witness_S = false; $\left(S_{a}\right)$

//@ ghost boolean witness_L = false; $\left(L_{a}\right)$

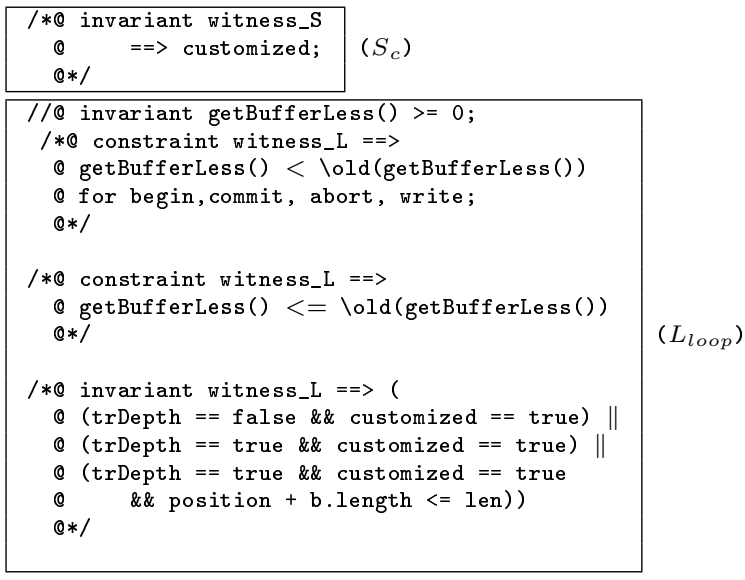

void storeData(int 1) \{

//ब set witness_S = true; $\left(S_{b}\right)$
$/ / \mathbb{Q}$ set witness_L $\left.=! \operatorname{trDepth} ;\left(L_{c}\right)\right\}$

void $\operatorname{begin}()\{$

Exception e1;

try \{ $\left(L_{b}\right)$

$\operatorname{try}\{$

$/ / \mathbb{Q}$ set witness_L $=$ !trDepth; $\left(L_{c}\right)$

\}

catch (Exception e) \{

e1 $=e$

\} \}

finally \{

if $(\mathrm{e} 1==\mathrm{null})$ \{

\}

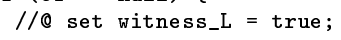

$\left(L_{b}\right)$

else \{

throw e1;

\}

void commit() \{

//ब set witness_L = !trDepth; $\left(L_{c}\right)$ \} void write (byte b)

//@ set witness_L = !trDepth; $\left.\left(L_{c}\right)\right\}$ void byte[] /*@ pure $\mathbb{Q} * /$ getStatus()\{ ... $\}$

Figure 5: Buffer with generated annotations 


\section{List of Tables}

1 Results for temporal properties verification $\ldots \ldots \ldots \ldots \ldots$ 


\begin{tabular}{|l|c|c|c|}
\hline $\begin{array}{l}\text { Example } \\
\text { Name }\end{array}$ & $\begin{array}{c}\text { Number } \\
\text { of temporal } \\
\text { properties } \\
\text { to verify }\end{array}$ & $\begin{array}{c}\text { Number } \\
\text { of generated } \\
\text { annotation } \\
\text { lines }\end{array}$ & $\begin{array}{c}\text { Number } \\
\text { of POs } \\
\text { (automatically } \\
\text { proved) }\end{array}$ \\
\hline TransactionSystem & 2 & 18 & $92(91)$ \\
\hline AtmTransaction & 2 & 21 & $171(171)$ \\
\hline Electronic Purse (Demoney) & 2 & 25 & $14(12)$ \\
\hline
\end{tabular}

Table 1: Results for temporal properties verification 